\title{
Spin-flip transitions induced by time-dependent electric fields in surfaces with strong spin-orbit interaction
}

\author{
Julen Ibañez-Azpiroz ${ }^{1,2}$, Asier Eiguren ${ }^{1,2}$, Eugene Ya. Sherman ${ }^{3,4}$, Aitor Bergara ${ }^{1,2,5}$ \\ ${ }^{1}$ Materia Kondentsatuaren Fisika Saila, University of Basque Country UPV-EHU 48080 Bilbao, Euskal Herria, Spain \\ ${ }^{2}$ Donostia International Physics Center (DIPC), \\ Paseo Manuel de Lardizabal 4, 20018 Donostia/San Sebastian, Spain \\ ${ }^{3}$ Department of Physical Chemistry University of Basque Country UPV-EHU 48080 Bilbao, Euskal Herria, Spain \\ ${ }^{4}$ IKERBASQUE Basque Foundation for Science, 48011 Bilbao, Bizkaia, Spain and \\ ${ }^{5}$ Centro de Física de Materiales CFM - Materials Physics Center MPC, Centro Mixto CSIC-UPV/EHU, \\ Edificio Korta, Avenida de Tolosa 72, 20018 Donostia, Basque Country, Spain
}

(Dated: June 24, 2018)

\begin{abstract}
We present a comprehensive theoretical investigation of the light absorption rate at the $\mathrm{Pb} / \mathrm{Ge}(111)-\beta \sqrt{3} \times \sqrt{3} R 30^{\circ}$ surface with strong spin-orbit coupling. Our calculations show that electron spin-flip transitions cause as much as $6 \%$ of the total light absorption, representing one order of magnitude enhancement over Rashba-like systems. Thus, it is demonstrated that a substantial part of the light irradiating this nominally non-magnetic surface is attenuated in spin flip processes. Remarkably, the spin-flip transition probability is structured in well defined hot spots within the Brillouin zone where the electron spin experiences a sudden $90^{\circ}$ rotation. This mechanism offers the possibility of an experimental approach to the spin-orbit phenomena by optical means.
\end{abstract}

PACS numbers: 71.18.+y, 73.20.r, 73.25.+i

Understanding electron spin transport and spin relaxation in quasi-two-dimensional (2D) systems is of a capital importance due to both, the fundamental reasons and the potential technological applications. The spin-orbit (SO) interaction is the most prominent relativistic effect leading to the fascinating phenomena recently observed in 2D systems, such as the quantum spin Hall effect [1, 2]. An experimentally accessible spin degree of freedom offers new route for the emergent field of spintronics, where the main features of charge dynamics are strongly influenced by the spin-related effects [3, 4]. The technical possibility of spin manipulation and control by means of an applied bias voltage is strongly supported by recent investigations on a variety of semiconducting alloy samples [5 7].

However, a strong SO coupling cannot be achieved in conventional semiconductors where the spin-splitting of the conduction electrons is limited to a few meV at most. As opposed, the relativistic effects completely dominate the electronic structure of many heavy-element surface materials and overlayers [8]. The reason resides on the breaking of the inversion symmetry and the associated gradient of the effective one-electron potential introduced at the interface. These effects lead to extraordinarily large (100-500 meV) spin-splittings among the so called Shockley-type surface states, as it was first observed in the free electron-like $\mathrm{Au}(111)$ noble metal surface [9]. This system is regarded as a prototype of the standard Rashba model [10, 11]. Considering the general form of the SO interaction in the non-relativistic limit,

$$
\hat{H}_{\mathrm{SO}}=-\frac{\hbar e}{4 m^{2} c^{2}} \hat{\boldsymbol{\sigma}} \cdot(\mathbf{k} \times \nabla V(\mathbf{r})),
$$

the Rashba SO coupling is recovered by taking the gradi- ent of the effective one-electron potential $V(\mathbf{r})$ as a constant and completely surface perpendicular. This model produces an entirely isotropic result, with a simple linear spin-splitting of the two spin sub-bands with chiral spin-polarization. However, many heavy-element adlayer or even clean surface materials, such as $\operatorname{Bi}(110)$ [12], $1 \mathrm{x} 1 \mathrm{H} / \mathrm{W}(110)$ 13], $\mathrm{Au} / \mathrm{Si}(557)$ 14, $\mathrm{Bi} / \mathrm{Si}(111)$ 15, 16 or $\mathrm{Tl} / \mathrm{Si}(111)$ [17 19] exhibit large anisotropic SO interaction, inducing complex spin textures that considerably deviate from the free electron-like picture of the Rashba model.

In this Letter, we investigate electrically induced spinflip excitations on the $\mathrm{Pb} / \mathrm{Ge}(111)-\beta \sqrt{3} \times \sqrt{3} R 30^{\circ}$ surface $(\sqrt{3} \mathrm{~Pb} / \mathrm{Ge}(111))$, considering the full spinor structure of the electron states within ab-initio density functional theory. The goal is to understand and quantify the striking mechanism leading to spin-flip transitions out of a purely electric perturbation in a non-magnetic material. This system presents two well defined spin-spit surface states crossing the Fermi level, while the bulk substrate remains semiconducting. Thus, we face a problem involving a completely spin-polarized 2D electron gas which is essentially decoupled from the bulk, i.e., an optimum scenario for studying surface spin-flip transitions [20]. Noteworthy, the strength of the SO interaction associated to the $\mathrm{Pb}$ atoms is two orders of magnitude larger $(\approx 300$ $\mathrm{meV}$ ) than in semiconductor quantum wells. Moreover, the $\mathrm{Pb}$ overlayer includes strong anisotropic gradients in the surface ionic potential, giving rise to a fast variation of the noncollinear spin-polarization in momentum space which critically enhances the spin-flip transition probability.

The spin manipulation in 2D systems [21] is accessible via electric-dipole spin resonance mechanism, which cou- 


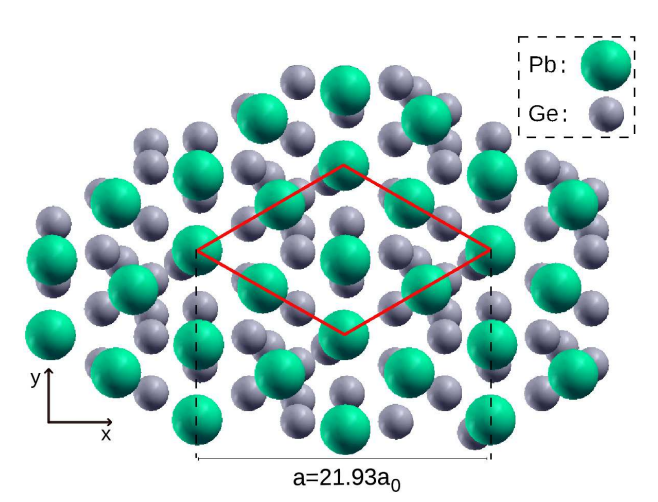

FIG. 1: (color online) Top view of the $\sqrt{3} \mathrm{~Pb} / \mathrm{Ge}(111)$ surface 25]. The small (gray) spheres symbolize the Ge substrate layers, whereas the big (green) spheres represent the $\mathrm{Pb}$ surface monolayer. The solid (red) parallelogram indicates surface unit cell.

ples the spin-dependent electron velocity to externally applied fields [22]. At surfaces, the different spin-spit sub-bands are connected by inter-band transitions that flip the electron spin [23]. The detailed analysis of these processes provides valuable information about the spindynamics of the system, thus making the first-principles approach imperative. Simplified tight-binding Rashbalike models, although very useful for understanding the basic physics [24], appear not to be realistic enough to account for material specific details which determine the electromagnetic response.

Let us consider the interaction of an electron with an external time-dependent electric field of frequency $\omega$. Within the electric dipole approximation valid for small momentum transfer $\mathbf{q} \rightarrow 0$, the interaction Hamiltonian is given by [22, 23]

$$
\hat{H}_{\mathrm{int}}(t)=-\frac{e}{c} \hat{\mathbf{v}} \cdot \mathbf{A}_{\mathrm{ext}}(t),
$$

where $\mathbf{A}_{\text {ext }}(t)=\mathbf{A}_{0} \cos \omega t$ is the external vector potential associated to the electric field $\mathbf{E}_{\text {ext }}(t)=\mathbf{E}_{0} \sin \omega t$ with $\mathbf{E}_{0}=\mathbf{A}_{0} \omega / c$, and $\hat{\mathbf{v}}$ is the electron velocity operator. In systems with SO coupling, besides the canonical contribution $\hat{\mathbf{p}} / m$, the velocity operator includes an additional spin-dependent term which emerges as the main responsible for the spin-flip transitions [23].

Within first order perturbation theory, the transition rate associated to spin-flip excitations between spin-spit surface states $S$ and $S^{\prime}$ due to photon absorption (term $\left.\mathbf{A}_{0} e^{-i \omega \cdot t} / 2\right)$ can be derived from the Fermi's golden rule,

$$
\begin{aligned}
\gamma_{S S^{\prime}}(\omega) & =\frac{2 \pi}{\hbar} \int_{S B Z}\left(f\left(\epsilon_{S \mathbf{k}}\right)-f\left(\epsilon_{S^{\prime} \mathbf{k}}\right)\right) \\
& \left|M_{S S^{\prime}}(\mathbf{k})\right|^{2} \delta\left(\epsilon_{S \mathbf{k}}-\epsilon_{S^{\prime} \mathbf{k}}-\hbar \omega\right) \frac{d^{2} k}{(2 \pi)^{2}}
\end{aligned}
$$

The integral is taken over the surface Brillouin zone (SBZ), $f\left(\epsilon_{i, \mathbf{k}}\right)$ and $\epsilon_{i, \mathbf{k}}$ represent the Fermi-Dirac distribution and a surface state eigenvalue, respectively, and

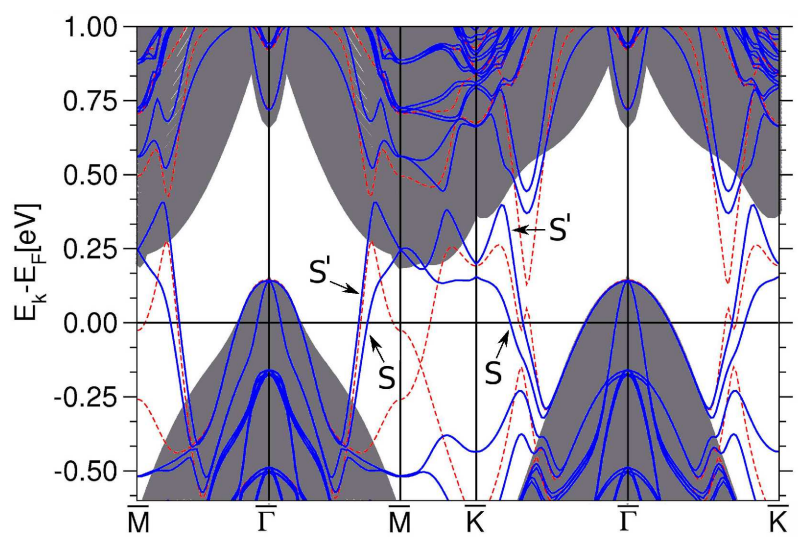

FIG. 2: (color online) Electron band structure of the $\sqrt{3} \mathrm{~Pb} / \mathrm{Ge}(111)$ surface. The scalar and fully relativistic bands are represented by dashed (red) and solid thick (blue) lines, respectively. The continuous background is the bulk band projection. The fully relativistic metallic surface states are labeled as $S$ and $S^{\prime}$.

$M_{S S^{\prime}}(\mathbf{k})$ is the interband matrix element

$$
M_{S S^{\prime}}(\mathbf{k})=-\frac{e \mathbf{A}_{0}}{2 c} \cdot\left\langle\Psi_{S \mathbf{k}}|\hat{\mathbf{v}}| \Psi_{S^{\prime} \mathbf{k}}\right\rangle
$$

Above, $\Psi_{i \mathbf{k}}(\mathbf{r})$ denotes the single-particle Bloch spinor wave function associated to a surface state.

Due to the inherent phase indeterminacy of the Bloch states in $\mathbf{k}$-space, the matrix elements of the velocity operator require a special treatment 26]. Following the approach presented in Ref. 27], we expressed $M_{S S^{\prime}}(\mathbf{k})$ in Eq. 4 in terms of the so called maximally localized Wannier functions 28]. In this way, the matrix elements entering Eq. 3 are maximally smooth and thus suitable for any interpolation procedure within the Brillouin zone. The Wannier functions were generated considering the entire structure of ab-initio spinor wave functions obtained within the noncollinear DFT formalism [2931]. The exchange-correlation energy was approximated by the standard LDA-PZ parametrization 32] and the $2 \times 2$ norm-conserving fully relativistic pseudopotential approach [31, 33]. The ground state self-consistent cycle was performed considering the usual Monckhorst-Pack mesh corresponding to a $27 \times 27$ grid. We employed a very fine $200 \times 200$ mesh considering the standard Wannier interpolation procedure for all the ingredients entering Eq. 3 27, 28] in order to reliably account for the details close to the Fermi level [27].

The $\sqrt{3} \mathrm{~Pb} / \mathrm{Ge}(111)$ surface (Fig. 1) was simulated by a repeated slab technique containing 14 Ge layers. The $\mathrm{Pb}$ monolayer was included only in one side of the slab, while the other (bare) Ge(111) surface was covered by a hydrogen adlayer in order to saturate the dangling bonds. We also analyzed the $\mathrm{Au}(111)$ metal surface considering a $22 \mathrm{Au}$ layer slab. In both systems, a full geometry optimization was performed until all atomic forces exerted 


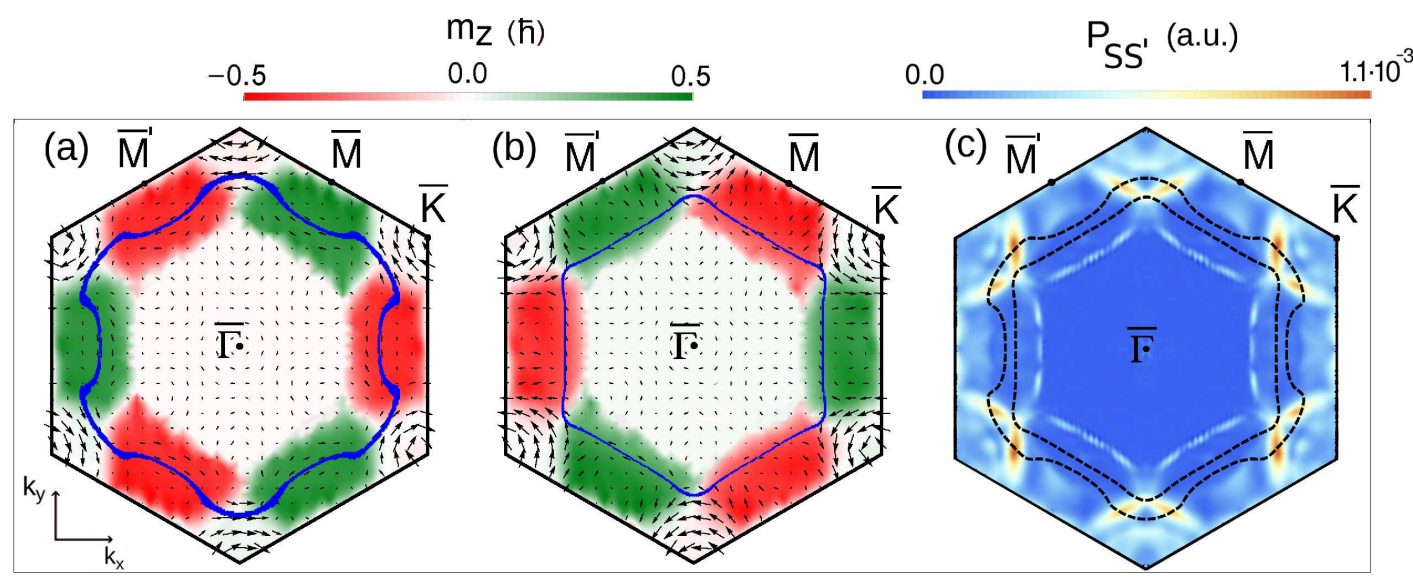

FIG. 3: (color online) (a) and (b) Momentum dependent spin-polarization structure associated to the $S$ and $S^{\prime}$ surface states, respectively. Arrows represent the in-plane spin-polarization component, whereas the background indicates the surface perpendicular component of the magnetization, $m_{z}(\mathbf{k})$. The Fermi surface of each state is indicated by solid (blue) lines. (c) Spin-flip transition probability associated to the $S$ and $S^{\prime}$ surface states for R-circularly polarized light.

on individual atoms were negligibly small $\left(<10^{-4}\right.$ Ry a.u. $\left.{ }^{-1}\right)$.

Fig. 2 shows the calculated band structure of the $\mathrm{Pb} / \mathrm{Ge}(111) \sqrt{3}$ surface. While the scalar relativistic calculation shows a single spin-degenerate surface band crossing the Fermi level, fully relativistic calculations present two spin-polarized surface bands labeled as $S$ and $S^{\prime}$. The SO interaction has a huge impact on the electron structure close to the Fermi level, such that this term cannot be treated perturbatively. Its contribution is even more important than some non-relativistic DFT terms such as the exchange-correlation energy. In this context, the SO interaction completely determines the metallic character of the $S$ and $S^{\prime}$ surface states. These exist as surface states only outside the area close to the $\bar{\Gamma}$ point, where $S$ and $S^{\prime}$ become resonances entering the bulk projection (continuum in Fig. 2). Outside this region, the splitting is in overall of the order of $100 \mathrm{meV}$, reaching a maximum of $300 \mathrm{meV}$ near the high symmetry point $\bar{M}$. The calculated Fermi wave vectors along the high symmetry direction $\overline{\Gamma M}, k_{F}^{S} \simeq 0.41 \AA^{-1}$ and $k_{F}^{S^{\prime}} \simeq 0.37 \AA^{-1}$, are in very good agreement with recent ARPES experiments reporting $k_{F}^{S}=0.40 \AA^{-1}$ and $k_{F}^{S^{\prime}}=0.36 \AA^{-1}[20]$.

A characteristic feature emerging from the SO interaction at surfaces is the momentum-dependent spin polarization:

$$
\boldsymbol{m}_{n}(\mathbf{k})=\int \Psi_{n \mathbf{k}}^{*}(\mathbf{r}) \hat{\boldsymbol{\sigma}} \Psi_{n \mathbf{k}}(\mathbf{r}) d^{3} r
$$

where $n$ is the band index. In Figs. $3 \mathrm{a}$ and $3 \mathrm{~b}$ we present the calculated spin-polarization for the $S$ and $S^{\prime}$ surface states in the entire Brillouin zone. These figures demonstrate that the $S$ and $S^{\prime}$ states are spin-polarized in almost the opposite direction, in agreement with recent spin-resolved ARPES measurements [20]. The negligible spin-polarization around $\bar{\Gamma}$ is consistent with the overlap of the surface bands with the bulk projection (see Fig. 2). In this area, the electron states become resonances with a large penetration, thus any surface effect such as the enhancement of the SO interaction is almost completely absent.

The anisotropic character of the SO interaction is evidenced by the highly noncollinear structure of the calculated spin-polarization for $S$ and $S^{\prime}$. On one hand, we observe that the spin of each surface state is mainly polarized along the surface perpendicular direction, a phenomenon that extends to a significant area around the high symmetry points $\overline{\mathrm{M}}$ and $\overline{\mathrm{M}^{\prime}}$. Such an important contribution of the out-of-plane magnetization is a consequence of the strong in-plane gradients of the ionic potential, as reported in the $\mathrm{Tl} / \mathrm{Si}(111)$ surface 17 19]. On the other hand, our calculations further identify an important area of almost pure in-plane circular spinpolarization for each state around high symmetry point $\overline{\mathrm{K}}$.

Fig. 3k shows the results for the calculated spin-flip transition probability $P_{S S^{\prime}}(\mathbf{k}) \equiv\left|M_{S S^{\prime}}(\mathbf{k})\right|^{2} /\left|\mathbf{A}_{0}\right|^{2}$ for an R-circularly polarized external field, $\mathbf{A}_{0}=A_{0}(\hat{\mathbf{x}}+$ $i \hat{\mathbf{y}}) / \sqrt{2}$. Results for other polarizations, although being slightly different, do not substantially modify our general conclusions. We deduce from Fig. 35 that the spin-flip transition probability is negligible around the $\bar{\Gamma}$ point. This is consistent with Eq. [4 since the surface states are spin-degenerate here, 27] therefore SO driven effects such as the spin-flip excitations are weak. On the other hand, the most important message of Fig. 3r is the extreme localization of the spin-flip transition probability in hot spots close to the high symmetry point $\overline{\mathrm{K}}$. A careful comparison of Figs. 3 and 3 b with Fig. 3reveals that the hot spots are localized in the boundaries separating the surface-perpendicular and surface-parallel 
spin-polarized regions in the Brillouin zone. This feature is fully consistent with Eq. 4 since the velocity operator introduces a k-space derivative [26, 27] which effectively measures the variation of the entire wavefunction and the spin-polarization through Eq. 5] Noteworthy, this singular effect is completely absent in Rashba-like systems exhibiting a smooth behavior of the spin-polarization 9 11].

The light absorption rate associated to surface spin-flip excitations is given by the following expression,

$$
\Omega_{S S^{\prime}}(\omega)=\frac{\hbar \omega \cdot \gamma_{S S^{\prime}}(\omega)}{W}
$$

Above, $\gamma_{S S^{\prime}}(\omega)$ is the spin-flip transition rate (Eq. 3), $\hbar \omega$ the energy of the external field and $W=c\left|E_{0}\right|^{2} / 8 \pi$, the incident optical power per unit area.

Fig. 4 illustrates the calculated absorption rate associated to the spin-spit surface states of the $\sqrt{3} \mathrm{~Pb} / \mathrm{Ge}(111)$ and the Rashba-like $\mathrm{Au}(111)$ surfaces. For $\sqrt{3} \mathrm{~Pb} / \mathrm{Ge}(111)$, the absorption spectrum is bounded in the $0.1-0.3 \mathrm{eV}$ energy range, corresponding approximately to the spin-splitting at the Fermi level. At this surface, the spectrum presents a prominent peak close to $0.17 \mathrm{eV}$, where the spin-flip absorption rate reaches a remarkable maximum value of $6 \%$.

This result demonstrates that a significant part of the incoming radiation is dissipated exclusively in the spinflip phenomena. The SO coupling making the orbital and spin degrees of freedom interrelated causes a question on the angular momentum conservation. As the absorption of circularly polarized light induces a net transfer of angular momentum, the resulting magnetization of the surface states can produce a corresponding angula momentum reservoir in a spin-orbit coupled system. Due to the anisotropy of the spin-polarization (see Fig. 3), the absorption spectrum exhibits a substantial variation as a function of the light polarization. As an example, close to $0.25 \mathrm{eV}$ the spin-flip absorption rate for $\mathrm{L}$ polarized light is approximately three times stronger than for $\mathrm{R}$ polarized light. In contrast, we find that the absorption spectrum of $\mathrm{Au}(111)$ is practically polarization independent. The reason is that this system exhibits an almost perfect isotropy, as assumed in the Rashba model. The magnitude of the spin-flip contribution for $\mathrm{Au}(111)$ is relatively weak $(0.8 \%$ maximum). Thus, the spin-flip absorption in $\sqrt{3} \mathrm{~Pb} / \mathrm{Ge}(111)$ is one order of magnitude stronger in comparison to $\mathrm{Au}(111)$.

It is noteworthy that the bare spin-flip contribution to the absorption rate in $\sqrt{3} \mathrm{~Pb} / \mathrm{Ge}(111)$ is around three times stronger than the total absorption of a graphene layer $(2.3 \%)$, where the electron spin does not play any significant role 34, 35. Thus, the a priori weaker relativistic SO interaction in $\sqrt{3} \mathrm{~Pb} / \mathrm{Ge}(111)$ exceeds the effect of the usually predominant non-relativistic terms such as the electric dipole mechanism. The reason why the spin-flip contribution in this system is so important

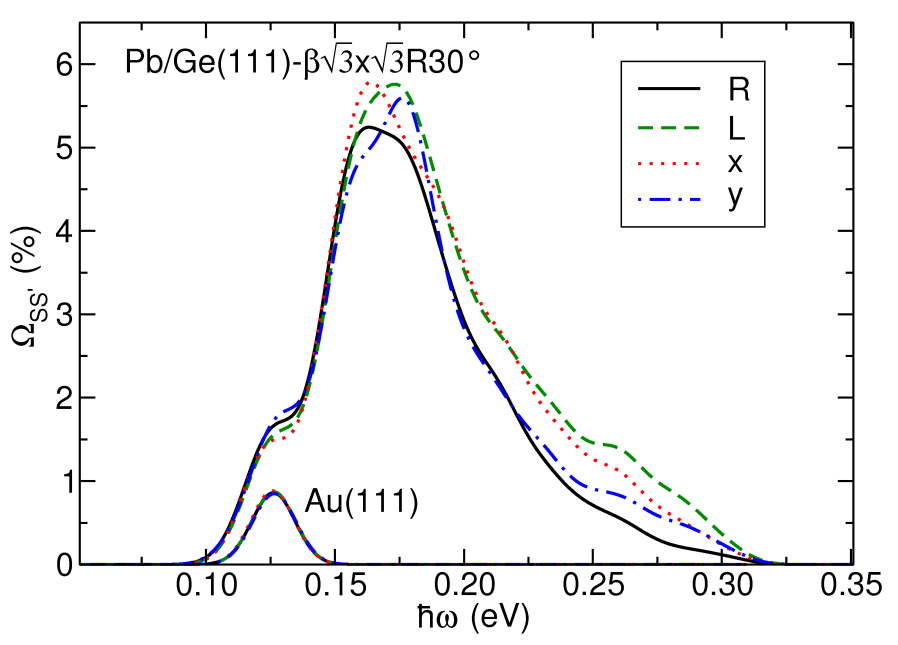

FIG. 4: (color online) Calculated spin-flip absorption rate in $\sqrt{3} \mathrm{~Pb} / \mathrm{Ge}(111)$ and $\mathrm{Au}(111)$. Solid (black), dashed (green), dotted (red) and dashed-doted (blue) lines represent the results corresponding to the $\mathrm{R}$ and $\mathrm{L}$ circularly polarized and $x$ and $y$ linearly polarized light, respectively. Note that the absorption rate for $\sqrt{3} \mathrm{~Pb} / \mathrm{Ge}(111)$ depends on the light polarization, while in $\mathrm{Au}(111)$ it is practically polarizationindependent.

is that the hot spot matrix elements (Fig. 3r) lie just inside the Brillouin zone area where the $S$ state is occupied and $S^{\prime}$ state remains empty. In this way, the Fermi occupation factors allow electron transitions precisely where the matrix elements are maximal. From the discussion above we can conclude that a large anomalous feature associated to the enhanced spin-flip excitation mechanism should be accessible by infrared optical spectroscopy in the $0.1-0.3 \mathrm{eV}$ energy range.

In summary, we investigate the role of the spinorbit interaction on the light absorption rate of the $\sqrt{3} \mathrm{~Pb} / \mathrm{Ge}(111)$ surface. Our calculations incorporate the full spinor wave function structure from first-principles, making use of a precise integration procedure through a Wannier interpolation scheme for the spin-flip matrix elements. We find that a substantial part of the lowenergy absorption spectrum is dominated exclusively by the spin-flip excitations associated to the spin-polarized surface states. Noteworthy, these transitions capture as much as $6 \%$ of the total incident power, representing an enhancement of one order of magnitude in comparison to the Rashba-like prototype $\mathrm{Au}(111)$ surface. The origin of such a huge absorption rate is closely related to the strong anisotropy exhibited by the spin-polarization structure connected to the surface states. As demonstrated in this Letter, a clear fingerprint of the spin-flip absorption mechanism should be accessible in the optical range, posing a challenge for further experimental work.

We acknowledge fruitful discussions with B. Rousseau, I. Errea, A. Kuzmenko and R. Winkler. This work was supported by the UPV/EHU (Grant No. IT-366-07 and 
program UFI 11/55), the Spanish Ministry of Science and Innovation (Grants No. FIS2010-19609-C02-00 and No. FIS2009-12773-C02-01), and the Basque Government (Grant No. IT472-10). The authors also acknowledge the Donostia International Physics Center (DIPC) for providing the computer facilities.

[1] C. L. Kane and E. J. Mele, Phys. Rev. Lett. 95, 146802 (2005).

[2] S. Zhang and Z. Yang, Phys. Rev. Lett. 94, 066602 (2005).

[3] J. I. Pascual, G. Bihlmayer, Y. M. Koroteev, H.-P. Rust, G. Ceballos, M. Hansmann, K. Horn, E. V. Chulkov, S. Blügel, P. M. Echenique, et al., Phys. Rev. Lett. 93, 196802 (2004).

[4] A. Strozecka, A. Eiguren, and J. I. Pascual, Phys. Rev. Lett. 107, 186805 (2011).

[5] J. B. Miller, D. M. Zumbühl, C. M. Marcus, Y. B. Lyanda-Geller, D. Goldhaber-Gordon, K. Campman, and A. C. Gossard, Phys. Rev. Lett. 90, 076807 (2003).

[6] M. A. Leontiadou, K. L. Litvinenko, A. M. Gilbertson, C. R. Pidgeon, W. R. Branford, L. F. Cohen, M. Fearn, T. Ashley, M. T. Emeny, B. N. Murdin, et al., Journal of Physics: Condensed Matter 23, 035801 (2011).

[7] O. Z. Karimov, G. H. John, R. T. Harley, W. H. Lau, M. E. Flatté, M. Henini, and R. Airey, Phys. Rev. Lett. 91, 246601 (2003).

[8] M. Heide, G. Bihlmayer, P. Mavropoulos, A. Bringer, and S. Blügel, Psi-k Newsletter 78 (2006).

[9] S. LaShell, B. A. McDougall, and E. Jensen, Phys. Rev. Lett. 77, 3419 (1996).

[10] E. I. Rashba, Sov. Phys. Solid State 2, 1109 (1960).

[11] E. I. Rashba and Y. A. Bychkov, JETP Lett. 39, 79 (1984).

[12] A. Strozecka, A. Eiguren, and J. I. Pascual, Phys. Rev. Lett. 107, 186805 (2011).

[13] A. Eiguren and C. Ambrosch-Draxl, New Jour. of Phys. 11, 013056 (2009).

[14] D. Sánchez-Portal, S. Riikonen, and R. M. Martin, Phys. Rev. Lett. 93, 146803 (2004).

[15] I. Gierz, T. Suzuki, E. Frantzeskakis, S. Pons, S. Ostanin, A. Ernst, J. Henk, M. Grioni, K. Kern, and C. R. Ast, Phys. Rev. Lett. 103, 046803 (2009).
[16] D. Khomitsky, Journal of Experimental and Theoretical Physics 114, 738 (2012).

[17] K. Sakamoto, T. Oda, A. Kimura, K. Miyamoto, M. Tsujikawa, A. Imai, N. Ueno, H. Namatame, M. Taniguchi, P. E. J. Eriksson, et al., Phys. Rev. Lett. 102, 096805 (2009).

[18] J. Ibañez Azpiroz, A. Eiguren, and A. Bergara, Phys. Rev. B 84, 125435 (2011).

[19] M.-H. Liu and C.-R. Chang, Phys. Rev. B 80, 241304 (2009)

[20] K. Yaji, Y. Ohtsubo, S. Hatta, H. Okuyama, K. Miyamoto, T. Okuda, A. Kimura, H. Namatame, M. Taniguchi, and T. Aruga, Nat. Commun. 1, 1 (2010).

[21] P. Tamarat, N. B. Manson, J. P. Harrison, R. L. McMurtrie, A. Nizovtsev, C. Santori, R. G. Beausoleil, P. Neumann, T. Gaebel, F. Jelezko, et al., New Journal of Physics 10, 045004 (2008).

[22] E. I. Rashba and A. L. Efros, Phys. Rev. Lett. 91, 126405 (2003).

[23] E. Y. Sherman, Phys. Rev. B 67, 161303 (2003).

[24] E. Frantzeskakis, S. Pons, and M. Grioni, Phys. Rev. B 82, 085440 (2010).

[25] K. Anton, Computational Materials Science 28, 155 (2003).

[26] E. I. Blount, Solid State Physics 13, 305 (1962).

[27] X. Wang, J. R. Yates, I. Souza, and D. Vanderbilt, Phys. Rev. B 74, 195118 (2006).

[28] I. Souza, N. Marzari, and D. Vanderbilt, Phys. Rev. B 65, 035109 (2001).

[29] A. A. Mostofi, J. R. Yates, Y. Lee, I. Souza, D. Vanderbilt, and N. Marzari, Computer Physics Communications 178, 685 (2008).

[30] P. Giannozzi, S. Baroni, N. Bonini, M. Calandra, R. Car, C. Cavazzoni, D. Ceresoli, G. L. Chiarotti, M. Cococcioni, I. Dabo, et al., Journal of Physics: Condensed Matter 21, 395502 (2009).

[31] A. DalCorso and A. MoscaConte, Phys. Rev. B 71, 115106 (2005).

[32] D. M. Ceperley and B. J. Alder, Phys. Rev. Lett. 45, 566 (1980).

[33] L. Kleinman and D. M. Bylander, Phys. Rev. Lett. 48, 1425 (1982).

[34] V. P. Gusynin, S. G. Sharapov, and J. P. Carbotte, Phys. Rev. Lett. 96, 256802 (2006).

[35] A. B. Kuzmenko, E. van Heumen, F. Carbone, and D. van der Marel, Phys. Rev. Lett. 100, 117401 (2008). 